\title{
Overexpression of TMOD1 is associated with enhanced regional lymph node metastasis in human oral cancer
}

\author{
TOSHIKAZU SUZUKI ${ }^{1}$, ATSUSHI KASAMATSU ${ }^{3}$, ISAO MIYAMOTO $^{1}$, TOMOAKI SAITO $^{1}$, MORIHIRO HIGO $^{3}$, \\ YOSUKE ENDO-SAKAMOTO $^{3}$, MASASHI SHIIBA ${ }^{2}$, HIDEKI TANZAWA ${ }^{1,2}$ and KATSUHIRO UZAWA ${ }^{1,3}$

\begin{abstract}
Departments of ${ }^{1}$ Oral Science and ${ }^{2}$ Medical Oncology, Graduate School of Medicine, Chiba University, Chuo-ku, Chiba;
${ }^{3}$ Department of Dentistry and Oral-Maxillofacial Surgery, Chiba University Hospital, Chuo-ku, Chiba 260-8670, Japan
\end{abstract}

Received October 20, 2015; Accepted November 27, 2015

DOI: $10.3892 /$ ijo.2015.3305

\begin{abstract}
Tropomodulin1 (TMOD1), which regulates the length and depolymerization of actin filaments by binding to the pointed end of the actin filament, has been reported to be a powerful diagnostic marker for ALK-negative anaplastic large-cell lymphoma; however, little is known about the relevance of TMOD1 in the behavior of oral squamous cell carcinoma (OSCC). We evaluated TMOD1 expression in OSCC-derived cell lines and primary OSCC samples $(n=200)$ using quantitative reverse transcriptase-polymerase chain reaction, immunoblotting and semi-quantitative immunohistochemistry. We also analyzed the clinical correlation between TMOD1 expression status and clinical parameters in patients with OSCC and performed a prospective study using 40 primary OSCC samples. TMOD1 expression was upregulated significantly $(\mathrm{P}<0.05)$ in OSCC in vitro and in vivo compared with normal counterparts. TMOD1 expression also was correlated significantly $(\mathrm{P}=0.0199$ and $\mathrm{P}=0.0064$, respectively) with regional lymph node metastasis (RLNM) and 5-year survival rates. This prospective study also showed that high TMOD1 expression was seen in $12(75 \%)$ of 16 cases in RLNM-positive patients and 9 (37.5\%) of 24 cases in RLNM-negative patients. The current data provide the first evidence that TMOD1 expression is a critical biomarker for RLNM and prognosis of patients with OSCC.
\end{abstract}

\section{Introduction}

Oral squamous cell carcinoma (OSCC) is a frequently occurring neoplasm that is usually aggressive and has a poor

Correspondence to: Dr Katsuhiro Uzawa, Department of Oral Science, Graduate School of Medicine, Chiba University, 1-8-1 Inohana, Chuo-ku, Chiba 260-8670, Japan

E-mail: uzawak@faculty.chiba-u.jp

Dr Atsushi Kasamatsu, Department of Dentistry and OralMaxillofacial Surgery, Chiba University Hospital, 1-8-1 Inohana, Chuo-ku, Chiba 260-8670, Japan

E-mail:kasamatsua@faculty.chiba-u.jp

Key words: tropomodulin1, oral squamous cell carcinoma, metastasis prognosis (1). OSCC accounts for $>50 \%$ of all head and neck SCC. The prognosis in advanced cases is poor, and the 5-year survival rates of OSCC are below $50 \%(2,3)$. The 5-year survival rate is $90 \%$ for patients without metastasis, but $<40 \%$ for patients with metastasis, suggesting that the regional lymph node metastasis (RLNM) is one of the most adverse prognostic factors (4-10). However, the mechanisms of metastasis are poorly understood (11). Therefore, molecular changes in a number of oncogenes and tumor suppressor genes associated with development of OSCC may be important clues for preventing this disease, and elucidating the molecular mechanisms involved in cancer metastasis is needed $(3,4)$.

The tropomodulin family (TMOD1-4) is expressed differentially in a tissue-specific manner and is involved in regulating actin filament architecture in diverse cellular types (12). TMOD1-4 are 70\% similar in amino acid sequence with different expression profiles (13) and contain an N-terminal unstructured domain and a C-terminal domain consisting of 5 leucine-rich repeat motifs $(14,15)$. TMOD1-4 inhibit elongation and depolymerization of actin filaments by binding to the pointed end of the actin filament (16-18). Among them, TMOD1 has two actin-binding regions and two tropomyosinbinding regions (19-22).

Recent studies have reported that TMOD1 is a diagnostic marker for triple-negative breast cancers and ALK-negative anaplastic large-cell lymphoma $(23,24)$; however, the role of TMOD1 in OSCC remains unknown. We present the results of measurements of TMOD1 levels in OSCC that are clinically and functionally linked to RLNM.

\section{Materials and methods}

Ethics statement. The Ethics Committee of the Graduate School of Medicine, Chiba University, Chiba, Japan (approval number, 236) approved the study protocol, which was performed in accordance with the tenets of the Declaration of Helsinki. All patients provided written informed consent.

OSCC-derived cell lines and tissue specimens. Human OSCC-derived cell lines (HSC-2, HSC-3, HSC-4, Sa3, Ca9-22, Ho-1-u-1, Ho-1-N-1, KOSC-2 and SAS) were obtained from the Human Science Research Resources Bank (Osaka, Japan) or the RIKEN BioResource Center (Ibaraki, Japan) 
Table I. Clinical classification in OSCCs from 200 patients.

\begin{tabular}{lrc}
\hline Variables & No. of patients & $(\%)$ \\
\hline Age at surgery (years) & & \\
$<70$ & 89 & 55.5 \\
$\geq 70$ & & 44.5 \\
Gender & 132 & 66 \\
Male & 68 & 34 \\
Female & & \\
T-primary tumor & 116 & 58 \\
T1 + T2 & 84 & 42 \\
T3 + T4 & & \\
N-regional lymph node & 120 & 60 \\
Negative & 80 & 40 \\
Positive & & \\
Histopathological type & 190 & 95 \\
Well and moderately differentiated & 10 & 5 \\
Poorly differentiated & & \\
Vascular invasion & 149 & 74.5 \\
Negative & 51 & 25.5 \\
Positive & & \\
\hline
\end{tabular}

through the National BioResource Project of the Ministry of Education, Culture, Sports, Science and Technology in Japan. Primary cultured human normal oral keratinocytes (HNOKs) were obtained from healthy oral mucosal epithelial specimens collected from young patients at Chiba University Hospital (25-28). All cells were grown in Dulbecco's modified Eagle's medium (Sigma-Aldrich, St. Louis, MO, USA) supplemented with $10 \%$ fetal bovine serum (FBS; Sigma-Aldrich) and 50 units/ml of penicillin and streptomycin (Sigma-Aldrich).

Two hundred primary OSCC specimens and patientmatched normal epithelial specimens were obtained during surgeries performed at Chiba University Hospital (Table I). The resected tissues were fixed in $20 \%$ buffered formaldehyde solution for pathological diagnosis and immunohistochemical (IHC) staining. We performed histopathological diagnosis of each OSCC sample according to the World Health Organization criteria at the Department of Pathology of Chiba University Hospital (29). The clinicopathological stages were determined based on the TNM classification of the International Union against Cancer (30).

mRNA expression analysis. Total RNA was isolated using TRIzol reagent (Invitrogen, Carlsbad, CA, USA), according to the manufacturer's instructions. cDNA was generated using ReverTra Ace qPCR RT Master Mix (Toyobo Life Science, Osaka, Japan) according to the manufacturer's instructions. Real-time quantitative reverse transcriptase-polymerase chain reaction (qRT-PCR) was performed in a $20-\mu 1$ reaction volume using the LightCycler 480 apparatus (Roche Diagnostics, Mannheim, Germany), according to the manufacturer's protocol. The general amplification conditions were performed as previously described (31-33). Primers and universal probes were designed using the Universal ProbeLibrary Assay Design Center (Roche Diagnostics), which specifies the most suitable set. The primer sequences used for qRT-PCR were: TMOD1, forward, 5'-AGCTGAGGACCCTGGAAAAT-3' and reverse, 5'-GCAGGCAGCAGTGCATTAT-3'; and universal probe \#42, and the glyceraldehyde-3-phosphate dehydrogenase (GAPDH), forward, 5'-CATCTCTGCCCCCTCTGCTGA-3' and reverse, 5'-GGATGACCTTGCCCACAGCCT-3'; and universal probe \#60. The transcript amount for TMOD1 was estimated from the respective standard curves and normalized to the GAPDH transcript amount determined in corresponding samples.

Immunoblotting analysis. The cells were washed three times with cold phosphate-buffered saline (PBS) and briefly centrifuged gently. The cellular pellets were incubated at $4^{\circ} \mathrm{C}$ for $30 \mathrm{~min}$ in a lysis buffer $(7 \mathrm{M}$ urea, $2 \mathrm{M}$ thiourea, $4 \% \mathrm{w} / \mathrm{v}$ CHAPS, and $10 \mathrm{mM}$ Tris; $\mathrm{pH}$ 7.4) with a proteinase inhibitor cocktail (Roche Diagnostics). The total protein concentration was measured using a dye-binding method based on the Bradford assay with Bio-Rad Protein Assay Dye Reagent Concentrate (Bio-Rad Laboratories, Hercules, CA, USA).

Protein extracts were electrophoresed on $4-12 \%$ Bis-Tris gel and transferred to nitrocellulose membranes (Invitrogen) and blocked for $1 \mathrm{~h}$ at room temperature with Blocking One (Nacalai Tesque, Inc., Kyoto, Japan). The membranes were washed three times with $0.1 \%$ Tween-20 in Tris-buffered saline (TBS-T) and incubated with affinity-purified rabbit antiTMOD1 monoclonal antibody (Santa Cruz Biotechnology, Santa Cruz, CA, USA) and mouse anti-GAPDH monoclonal antibody overnight at $4^{\circ} \mathrm{C}$. The membrane was washed with TBS-T and incubated with horseradish peroxidaseconjugated anti-rabbit or anti-mouse $\operatorname{IgG}$ as a secondary antibody (Promega Corp., Madison, WI, USA), for $1 \mathrm{~h}$ at room temperature. Finally, the membranes were detected using SuperSignal West Pico Chemiluminescent substrate (Thermo Fisher Scientific, Rockford, IL, USA), and immunoblotting was visualized by exposing the membranes to the ChemiDoc XRS Plus system (Bio-Rad Laboratories). The signal intensities were quantitated using the Image Lab system (Bio-Rad Laboratories). Densitometric TMOD1 protein data were normalized to GAPDH protein levels.

Semi-quantitative IHC. Semi-quantitative IHC (sq-IHC) of 4- $\mu \mathrm{m}$ sections of paraffin-embedded OSCC clinical specimens was performed. Briefly, after paraffinization, hydration, activation of antigen, hydrogen peroxide quenching, and blocking, the clinical sections were incubated with rabbit antiTMOD1 monoclonal antibody (Santa Cruz Biotechnology) at $4^{\circ} \mathrm{C}$ in a moist chamber overnight. Upon incubation with the primary antibody, the specimens were washed three times with PBS and treated with EnVision reagent (Dako, Carpinteria, CA, USA) followed by color development in 3,3'-diaminobenzidine tetrahydrochloride (Dako). The slides then were counterstained lightly with hematoxylin, dehydrated with ethanol, cleaned with xylene and mounted. To quantify the status of the TMOD1 protein expression in clinical samples, we used the sq-IHC scoring systems previously described (28,34-37). The mean percentages 

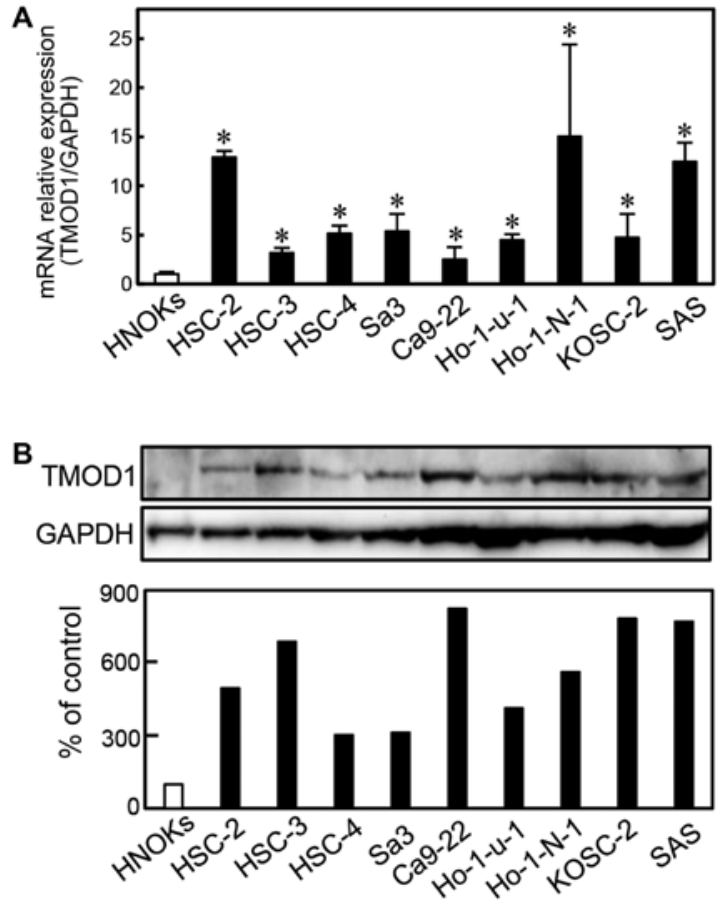

Figure 1. Upregulation of TMOD1 in OSCC-derived cell lines. (A) Quantification of TMOD1 mRNA expression in OSCC-derived cell lines by qRT-PCR analysis. Significant ( $\mathrm{P}<0.05$, Student's t-test) upregulation of TMOD1 mRNA is seen in 9 OSCC-derived cell lines compared with the HNOKs. Data are expressed as the mean \pm SEM of triplicate results (A) Immunoblotting analysis of TMOD1 protein in OSCC-derived cell lines. TMOD1 protein expression is upregulated in OSCC-derived cell lines compared with that in the HNOKs. Densitometric TMOD1 protein data are normalized to the GAPDH protein levels. The values are expressed as percentages of the HNOKs (B).

of positive tumoral cells were determined in at least three random fields in each section; the intensities of the TMOD1immunoreactions were scored as follows: $0+$, none; $1+$, weak; $2+$, moderate; and $3+$, intense. The staining intensity and the numbers were multiplied to produce a TMOD1 sq-IHC score. To determine the cut-off points of the TMOD1 sq-IHC scores, we analyzed the OSCCs sq-IHC scores of 200 patients using receiver operating characteristic (ROC) curves. Two independent pathologists from Chiba University Hospital, neither of whom had knowledge of the clinical status of the patients, made these judgments. To calculate the 5-year survival rate, we followed-up each patient, until death.

Prospective study. To evaluate the effect of the cut-off value from RLNM by ROC curve analysis, we performed a prospective study using 40 primary OSCC specimens at Chiba University Hospital. We randomly selected 40 primary OSCC specimens and analyzed the correlation between RLNM and TMOD1 expression using sq-IHC.

Statistical analysis. To compare the TMOD1 expression levels, statistical significance was evaluated using the Mann-Whitney $\mathrm{U}$ test. The relationships between the TMOD1 sq-IHC scores and clinicopathological profiles were evaluated using the Student's t-test and the Mann-Whitney U test. The 5-year survival rate was evaluated using the log-rank test. $\mathrm{P}<0.05$ was

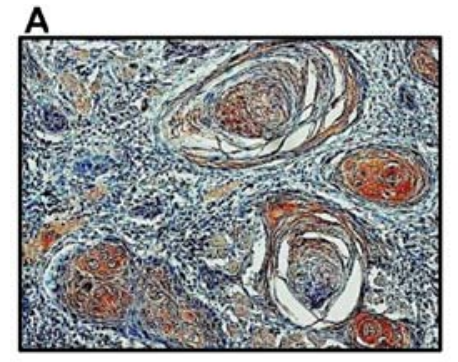

B
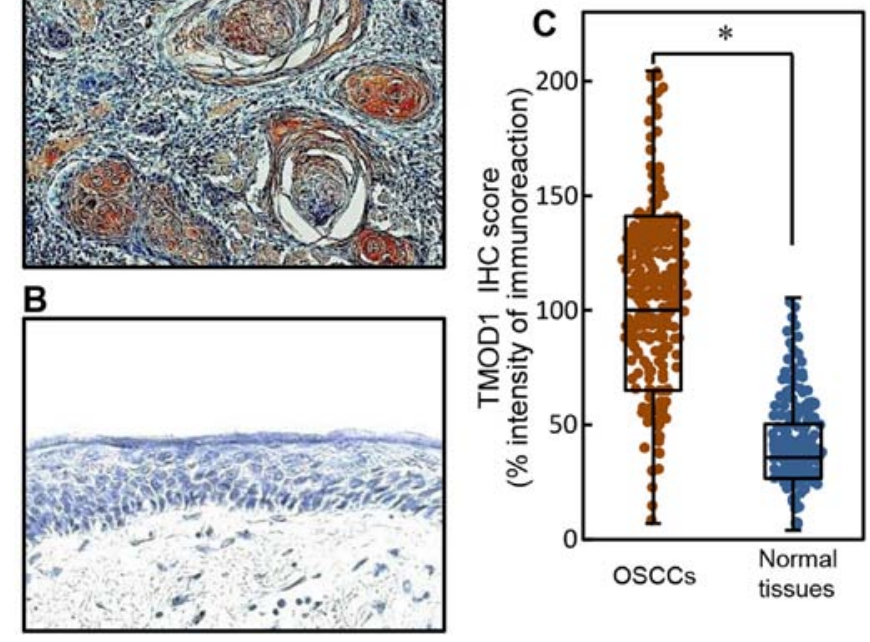

Figure 2. Evaluation of TMOD1 expression in primary OSCCs. Representative sq-IHC results of TMOD1 in primary OSCCs (A) and normal oral tissues (B). Original magnification, $x 200$. The status of TMOD1 protein expression in primary OSCCs $(n=200)$ and normal counterparts by the sq-IHC scoring system. The TMOD1 sq-IHC scores for OSCCs and normal oral tissues range from 204.44 to 7.00 (median, 100.00) and from 105.50 to 4.00 (median, 35.83), respectively. TMOD1 protein expression levels in OSCCs are significantly higher $\left({ }^{*} \mathrm{P}<0.05\right.$, Student's t-test) than in normal oral tissue $(C)$.

considered statistically significant. The data are expressed as the mean \pm the standard error of the mean.

\section{Results}

Upregulation of TMODI in OSCC-derived cell lines. To investigate the expression status of TMOD1, we performed qRT-PCR and immunoblotting analyses using 9 OSCCderived cell lines (HSC-2, HSC-3, HSC-4, Sa3, Ca9-22, Ho-1-u-1, Ho-1-N-1, KOSC-2 and SAS) and HNOKs. TMODI mRNA was upregulated significantly $(\mathrm{P}<0.05)$ in all OSCCderived cell lines compared with the HNOKs (Fig. 1A). We also performed immunoblotting analysis to investigate the TMOD1 protein expression in the OSCC-derived cell lines and the HNOKs (Fig. 1B). A significant increase in TMOD1 protein expression was seen in all OSCC-derived cell lines compared with the HNOKs.

Evaluation of TMOD1 expression in primary OSCCs. To investigate the expression status of TMOD1 in primary OSCCs and the relation to the clinicopathological characteristics, we analyzed the TMOD1 protein expression in primary OSCC specimens from 200 patients (Table I) using the sq-IHC scoring system. We showed representative IHC results for TMOD1 protein in primary OSCCs (Fig. 2A) and normal oral tissue (Fig. 2B). Strong TMOD1 immunoreactivity was detected in the cytoplasm of primary OSCCs; however, normal oral tissue showed almost negative immunostaining. The TMOD1 protein expression of primary OSCCs was significantly $(\mathrm{P}<0.05)$ higher than in normal tissue (Fig. $2 \mathrm{C})$. The TMOD1 sq-IHC scores in OSCCs and adjacent normal oral tissues ranged from 204.44 to 7.00 (median, 100.00) and from 105.50 to 4.00 (median, 35.83), respectively. 
A
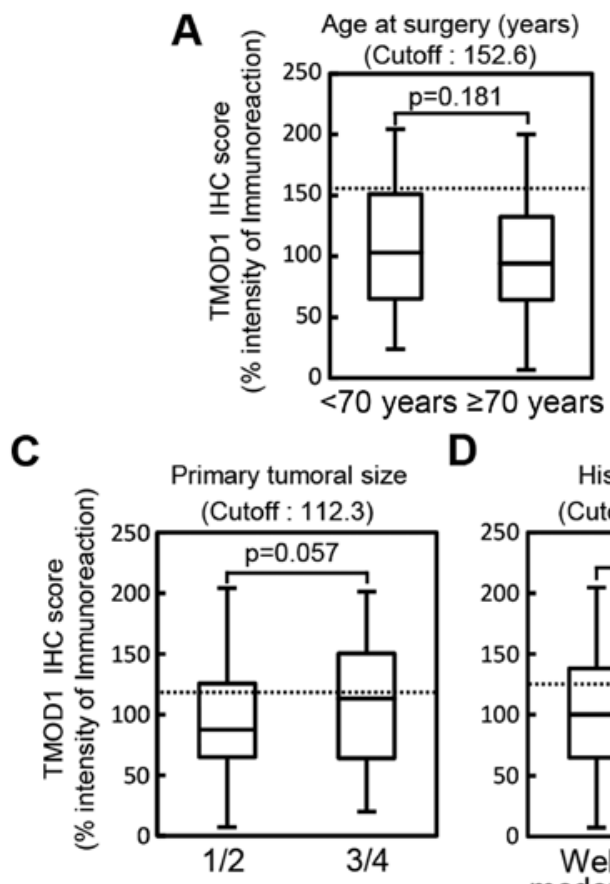

B

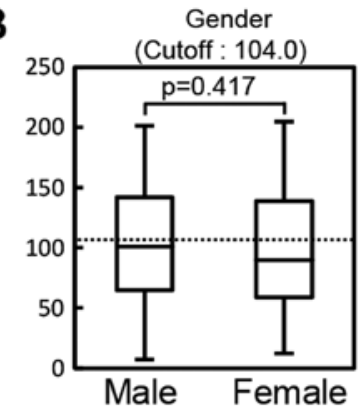

D

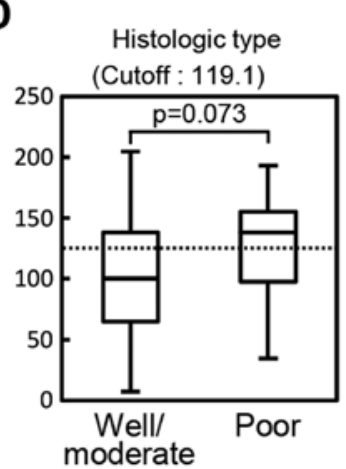

E

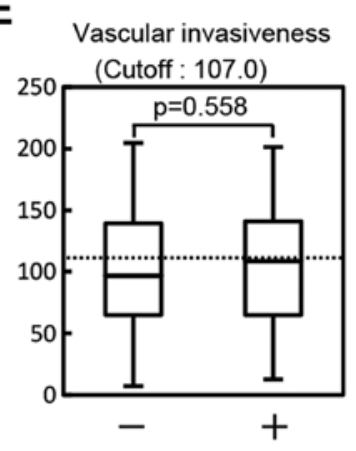

Figure 3. Evaluation of TMOD1 expression in primary OSCCs by various clinical parameters. The evaluation of TMOD1 expression based on the age at surgery shows that the optimal cut-off point in the ROC curve analysis is 152.6 (AUC, 0.556; 95\% CI, 0.476-0.636; sensitivity, 88.8\%; specificity, 25.5\%). The TMOD1 OSCCs sq-IHC scores in patients under 70 years of age and over 70 years of age range from 204.44 to 24.00 (median, 103.00) and 200.00 to 7.00 (median, 94.00), respectively. The TMOD1 protein expression in the primary OSCCs does not differ significantly ( $\mathrm{P}=0.18$, Student's $\mathrm{t}$-test) between the two age groups (A). Evaluation of TMOD1 expression in primary OSCCs by gender shows that the optimal cut-off point in the ROC curve analysis is 104.0 (AUC, 0.530; 95\% CI, 0.445-0.616; sensitivity, 47.7\%; specificity, 61.2\%). The TMOD1 OSCCs sq-IHC scores in males and females range from 201.17 to 7.00 (median, 101.00) and 204.44 to 12.50 (median, 89.92), respectively. The TMOD1 protein expression in the primary OSCCs does not differ significantly ( $\mathrm{p}=0.417$, Student's t-test) between males and females (B). Evaluation of TMOD1 expression by primary tumoral size shows that the optimal cut-off point in the ROC curve analysis is 112.3 (AUC, $0.578 ; 95 \%$ CI, 0.496-0.660; sensitivity, 70.7\%; specificity, 52.4\%). The TMOD1 OSCCs sq-IHC scores in T1/T2 and T3/T4 range from 204.44 to 7.00 (median, 87.46) and 201.67 to 20.00 (median, 113.13), respectively. The TMOD1 protein expression in the primary OSCCs does not differ significantly ( $\mathrm{P}=0.057$, Student's t-test) between T1/T2 and T3/T4 (C). Evaluation of TMOD1 expression in primary OSCCs by histologic type shows that the optimal cut-off point in the ROC curve analysis is 119.1 (AUC, 0.665 ; 95\% CI, 0.489-0.841; sensitivity, 68.3\%; specificity, 70.0\%). The TMOD1 OSCCs sq-IHC scores in well/moderately differentiated OSCCs and poorly differentiated OSCCs range from 204.44 to 7.00 (median, 100.00) and 193.17 to 34.50 (median, 138.14), respectively. The TMOD1 protein expression in the primary OSCCs does not differ significantly ( $\mathrm{P}=0.073$, Student's t-test) between well/moderately differentiated OSCCs and poorly differentiated OSCCs (D). Evaluation of TMOD1 expression in primary OSCCs by vascular invasiveness shows that the optimal cut-off point in the ROC curve analysis is 107.0 (AUC, 0.527; 95\% CI, 0.437-0.618; sensitivity, 60.4\%; specificity, 60.0\%). The TMOD1 OSCCs sq-IHC scores with/without vascular invasion range from 204.44 to 7.00 (median, 97.00) and 201.17 to 12.50 (median, 108.53), respectively. The TMOD1 protein expression in the primary OSCCs does not differ significantly ( $\mathrm{P}=0.558$, Student's t-test) with/without vascular invasion (E).
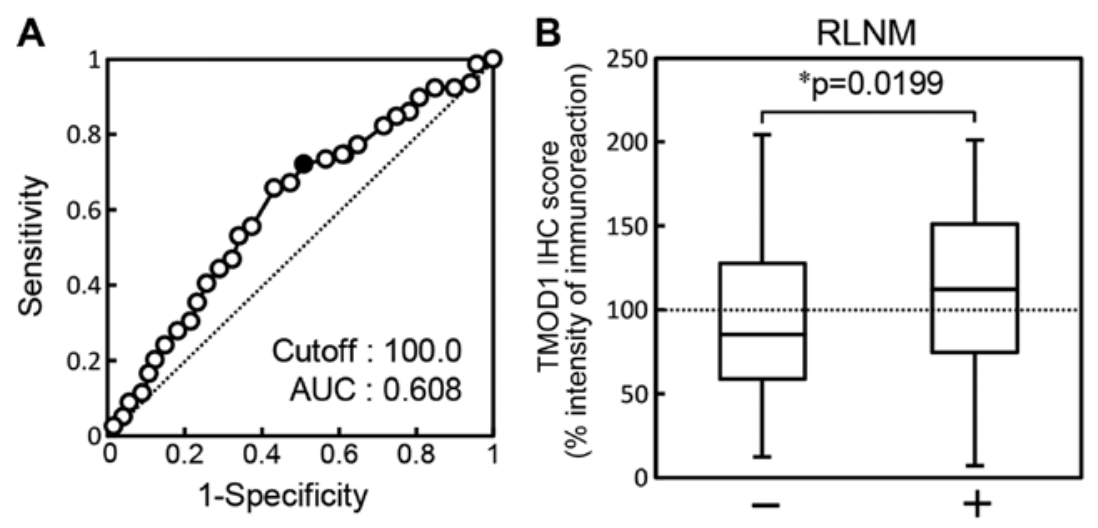

Figure 4. Evaluation of TMOD1 expression in primary OSCCs by RLNM. ROC curve analysis shows that the optimal cut-off point is 100.00 (AUC, 0.608 ; $95 \%$ CI, 0.527-0.688; sensitivity, 65.8\%; specificity, 57.5\%) (A). The TMOD1 OSCCs sq-IHC scores without RLNM and with RLNM range from 204.44 to 12.50 (median, 85.48) and from 201.17 to 7.00 (median, 112.16), respectively (B). The TMOD1 protein expression in the primary OSCCs with RLNM is significantly $(\mathrm{P}=0.0199$, Student's t-test) higher than those without RLNM.

Evaluation of TMOD1 expression in primary OSCCs by age at surgery, gender, primary tumoral size, histologic type and vascular invasiveness. We did not find differences between TMOD1 protein expression and the clinical parameters 


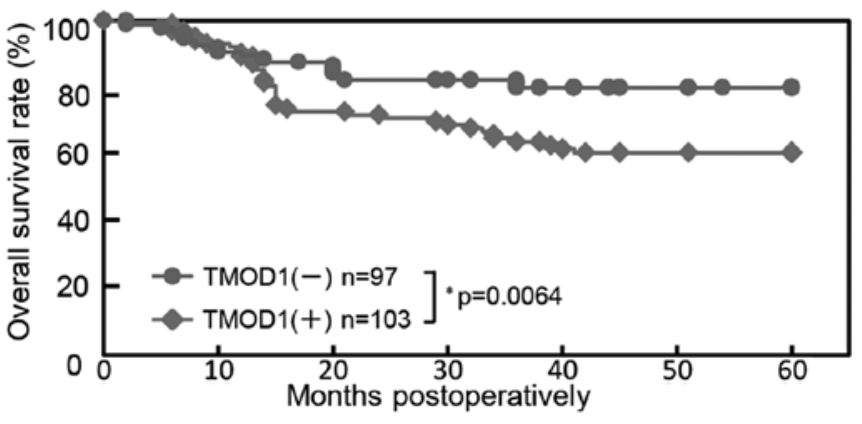

Figure 5. Evaluation of TMOD1 expression in primary OSCCs in patients with 5-year survival. Using the RLNM cut-off value from the ROC curve analysis, the TMOD1 expression level is significantly $(\mathrm{P}=0.0064$, log-rank test) correlated with the 5-year survival. The 5-year survival rates in the TMOD1-positive OSCCs $(\mathrm{n}=103)$ and the TMOD1-negative OSCCs $(\mathrm{n}=97)$ are 60.4 and $79.9 \%$, respectively.

Table II. Prospective study of TMOD1 expression in primary OSCCs from 40 patients.

\begin{tabular}{lrrr}
\hline & \multicolumn{2}{c}{$\operatorname{RLNM}(\mathrm{n}=40)$} & \\
\cline { 2 - 3 } Relative expression & \multicolumn{1}{c}{$(\%)$} & $+(\%)$ & P-value \\
\hline High TMOD1 & $9(37.5)$ & $12(75)$ & 0.027 \\
Low TMOD1 & $15(62.5)$ & $4(25)$ & \\
Total & $24(100)$ & $16(100)$ & \\
\hline
\end{tabular}

High TMOD1 expression is seen in 12 (75\%) of 16 RLNM-positive patients and $9(37.5 \%)$ of 24 RLNM-negative patients. Low TMOD1 expression is seen in 4 (25\%) of 16 RLNM-positive patients and 15 $(62.5 \%)$ of 24 RLNM-negative patients

(Fig. 3) (age at surgery, $\mathrm{P}=0.181$; gender, $\mathrm{P}=0.417$; primary tumoral size, $\mathrm{P}=0.057$; histologic type, $\mathrm{P}=0.073$; or vascular invasion, $\mathrm{P}=0.558$ ).

Evaluation of TMOD1 expression in primary OSCCs by $R L N M$. The ROC curve analysis showed that the area under the curve (AUC) was 0.608 [95\% confidence interval (CI), 0.527-0.688; sensitivity, 65.8\%; specificity, 57.5\%] and the cut-off value was 100.00 (Fig. 4A). The TMOD1 sq-IHC scores of the RLNM-negative patients and RLNM-positive patients ranged from 204.44 to 12.50 (median, 85.48) and from 201.17 to 7.00 (median, 112.16), respectively. TMOD1 protein expression of primary OSCCs with RLNM was significantly $(\mathrm{P}=0.0199)$ higher than without RLNM (Fig. 4B).

Evaluation of TMOD1 expression in primary OSCCs with 5-year survival. Using the cut-off value from RLNM from ROC curve analysis, the 5-year survival rates in the TMOD1positive OSCCs $(n=103)$ and the TMOD1-negative OSCCs $(n=97)$ were 60.4 and $79.9 \%$, respectively. The survival rates in the TMOD1-positive group were significantly $(\mathrm{P}=0.0064)$ lower than those in the TMOD1-negative group (Fig. 5).

Prospective study of TMOD1 expression in primary OSCCS. To determine if the cut-off value of the TMOD1 IHC scores from RLNM (Fig. 4) are useful as a clinical indicator, we prospectively assessed the correlation between RLNM and TMOD1 expression in 40 patients with OSCC. High TMOD1 expression was seen in 12 (75\%) of 16 RLNM-positive patients and 9 (37.5\%) of 24 RLNM-negative patients. Thus, TMOD1 expression was significantly $(\mathrm{P}=0.027)$ higher in the RLNMpositive patients (Table II).

\section{Discussion}

We found that TMOD1 was overexpressed frequently in OSCC in vitro and in vivo $(\mathrm{P}<0.05$; Figs. 1 and 2$)$, and that TMOD1 expression in RLNM-positive patients with OSCC was significantly $(\mathrm{P}<0.05)$ greater than in RLNM-negative patients (Fig. 4). In addition, the survival rates in the TMOD1-positive patients were significantly lower than in the TMOD1-negative patients (Fig. 5). In the prospective study, high TMOD1 expression was seen in 12 (75\%) of 16 RLNM-positive patients and 9 (37.5\%) of 24 RLNM-negative patients (Table II).

OSCCs are characterized by a high degree of local invasiveness and a high rate of RLNM in an early phase (38). A study reported recently that $37 \%$ of patients with OSCC had RLNM (39). The 5-year survival rate in RLNM-negative patients was $81 \%$, whereas that in RLNM-positive patients was $57 \%$ (39). Metastasis represents a highly organized, non-random, organspecific and multistep process (40). Although many molecules, such as integrins and matrix metalloproteinases (MMPs), play key roles in cancer cell invasiveness and metastasis (41-43), the precise factors and mechanisms affecting its preferred migration and invasion into the regional lymph nodes are poorly understood. Overexpression of TMOD1, a novel target of NF- $\kappa \mathrm{B}$, induces the translocation of $\beta$-catenin to nucleus, leading to activation of MMPs in triple-negative breast cancer samples (23). Since NF- $\kappa$ B signaling also relates to RLNM and tumor-induced lymphangiogenesis (44), our hypothesis is that TMOD1 may contribute to the cellular invasiveness and metastasis in OSCCs through the NF- $\mathrm{BB}$ signaling.

In conclusion, the current results indicated that TMOD1 is overexpressed frequently in human oral cancer. TMOD1 overexpression is associated with RLNM and the 5-year survival rate. The prospective study also confirmed the correlation between TMOD1 expression and RLNM. While further studies are needed to study the NF-кB-TMOD1 axis in the cancer microenvironment, TMOD1 overexpression may directly affect tumoral metastasis in OSCCs, and TMOD1 may be a critical biomarker of RLNM.

\section{Acknowledgements}

We thank Lynda C. Charters for editing this manuscript.

\section{References}

1. Severino P, Alvares AM, Michaluart P Jr, Okamoto OK, Nunes FD, Moreira-Filho CA and Tajara EH; Head and Neck Genome Project GENCAPO: Global gene expression profiling of oral cavity cancers suggests molecular heterogeneity within anatomic subsites. BMC Res Notes 1: 113, 2008.

2. Yao M, Epstein JB, Modi BJ, Pytynia KB, Mundt AJ and Feldman LE: Current surgical treatment of squamous cell carcinoma of the head and neck. Oral Oncol 43: 213-223, 2007. 
3. Casiglia $J$ and Woo SB: A comprehensive review of oral cancer. Gen Dent 49: 72-82, 2001.

4. Takes RP: Staging of the neck in patients with head and neck squamous cell cancer: Imaging techniques and biomarkers. Oral Oncol 40: 656-667, 2004.

5. Karatzanis AD, Waldfahrer F, Psychogios G, Hornung J, Zenk J, Velegrakis GA and Iro H: Resection margins and other prognostic factors regarding surgically treated glottic carcinomas. J Surg Oncol 101: 131-136, 2010

6. Fan S, Tang QL, Lin YJ, Chen WL, Li JS, Huang ZQ, Yang ZH, Wang YY, Zhang DM, Wang HJ, et al: A review of clinical and histological parameters associated with contralateral neck metastases in oral squamous cell carcinoma. Int J Oral Sci 3: 180-191, 2011.

7. Lea J, Bachar G, Sawka AM, Lakra DC, Gilbert RW, Irish JC, Brown DH, Gullane PJ and Goldstein DP: Metastases to level IIb in squamous cell carcinoma of the oral cavity: A systematic review and meta-analysis. Head Neck 32: 184-190, 2010.

8. Okura M, Aikawa T, Sawai NY, Iida S and Kogo M: Decision analysis and treatment threshold in a management for the NO neck of the oral cavity carcinoma. Oral Oncol 45: 908-911, 2009.

9. Greenberg JS, Fowler R, Gomez J, Mo V, Roberts D, El Naggar AK and Myers JN: Extent of extracapsular spread: A critical prognosticator in oral tongue cancer. Cancer 97: 1464-1470, 2003

10. Sano D and Myers JN: Metastasis of squamous cell carcinoma of the oral tongue. Cancer Metastasis Rev 26: 645-662, 2007.

11. Tanaka J, Irié T, Yamamoto G, Yasuhara R, Isobe T, Hokazono C, Tachikawa T, Kohno Y and Mishima K: ANGPTL4 regulates the metastatic potential of oral squamous cell carcinoma. J Oral Pathol Med 44: 126-133, 2015.

12. Lewis RA, Yamashiro S, Gokhin DS and Fowler VM: Functional effects of mutations in the tropomyosin-binding sites of tropomodulin1 and tropomodulin3. Cytoskeleton Hoboken 71: 395-411, 2014

13. Bliss KT, Tsukada T, Novak SM, Dorovkov MV, Shah SP, Nworu C, Kostyukova AS and Gregorio CC: Phosphorylation of tropomodulin1 contributes to the regulation of actin filament architecture in cardiac muscle. FASEB J 28: 3987-3995, 2014.

14. Krieger I, Kostyukova A, Yamashita A, Nitanai Y and Maéda Y: Crystal structure of the C-terminal half of tropomodulin and structural basis of actin filament pointed-end capping. Biophys J 83: 2716-2725, 2002.

15. Kostyukova A, Maeda K, Yamauchi E, Krieger I and Maéda Y: Domain structure of tropomodulin: Distinct properties of the N-terminal and C-terminal halves. Eur J Biochem 267: 6470-6475, 2000

16. Kostyukova AS: Tropomodulins and tropomodulin/tropomyosin interactions. Cell Mol Life Sci 65: 563-569, 2008.

17. Gregorio CC, Weber A, Bondad M, Pennise CR and Fowler VM: Requirement of pointed-end capping by tropomodulin to maintain actin filament length in embryonic chick cardiac myocytes. Nature 377: 83-86, 1995.

18. Tsukada T, Kotlyanskaya L, Huynh R, Desai B, Novak SM Kajava AV, Gregorio CC and Kostyukova AS: Identification of residues within tropomodulin-1 responsible for its localization at the pointed ends of the actin filaments in cardiac myocytes. J Biol Chem 286: 2194-2204, 2011.

19. Fowler VM, Greenfield NJ and Moyer J: Tropomodulin contains two actin filament pointed end-capping domains. J Biol Chem 278: 40000-40009, 2003.

20. Greenfield NJ, Kostyukova AS and Hitchcock-DeGregori SE: Structure and tropomyosin binding properties of the N-terminal capping domain of tropomodulin 1. Biophys J 88: 372-383, 2005.

21. Kostyukova AS, Choy A and Rapp BA: Tropomodulin binds two tropomyosins: A novel model for actin filament capping. Biochemistry 45: 12068-12075, 2006.

22. Kostyukova AS, Rapp BA, Choy A, Greenfield NJ and HitchcockDeGregori SE: Structural requirements of tropomodulin for tropomyosin binding and actin filament capping. Biochemistry 44: 4905-4910, 2005.

23. Ito-Kureha $\mathrm{T}$, Koshikawa N, Yamamoto M, Semba K, Yamaguchi N, Yamamoto T, Seiki M and Inoue J: Tropomodulin 1 expression driven by NF- $\mathrm{BB}$ enhances breast cancer growth. Cancer Res 75: 62-72, 2015.

24. Agnelli L, Mereu E, Pellegrino E, Limongi T, Kwee I, Bergaggio E, Ponzoni M, Zamò A, Iqbal J, Piccaluga PP, et al; European T-Cell Lymphoma Study Group: Identification of a 3 -gene model as a powerful diagnostic tool for the recognition of ALK-negative anaplastic large-cell lymphoma. Blood 120: 1274-1281, 2012
25. Kasamatsu A, Uzawa K, Nakashima D, Koike H, Shiiba M, Bukawa $\mathrm{H}$, Yokoe $\mathrm{H}$ and Tanzawa $\mathrm{H}$ : Galectin-9 as a regulator of cellular adhesion in human oral squamous cell carcinoma cell lines. Int J Mol Med 16: 269-273, 2005

26. Endo Y, Uzawa K, Mochida Y, Shiiba M, Bukawa H, Yokoe H and Tanzawa $\mathrm{H}$ : Sarcoendoplasmic reticulum $\mathrm{Ca}^{2+}$ ATPase type 2 downregulated in human oral squamous cell carcinoma. Int $\mathbf{J}$ Cancer 110: 225-231, 2004

27. Sakuma K, Kasamatsu A, Yamatoji M, Yamano Y, Fushimi K, Iyoda M, Ogoshi K, Shinozuka K, Ogawara K, Shiiba M, et al: Expression status of Zic family member 2 as a prognostic marker for oral squamous cell carcinoma. J Cancer Res Clin Oncol 136: 553-559, 2010.

28. Yamatoji M, Kasamatsu A, Kouzu Y, Koike H, Sakamoto Y, Ogawara K, Shiiba M, Tanzawa H and Uzawa K: Dermatopontin: A potential predictor for metastasis of human oral cancer. Int $J$ Cancer 130: 2903-2911, 2012.

29. Pindborg J, Reichart P, Smith C and Van der Waal I: Histological Typing of Cancer and Precancer of the Oral Mucosa. 2nd edition. Springer-Verlag, Berlin, 1997.

30. Sobin LH, Gospodarowicz MK and Wittekind C: TNM Classification of Malignant Tumours. 7th edition. John Wiley \& Sons, 2011.

31. Shimizu F, Shiiba M, Ogawara K, Kimura R, Minakawa Y, Baba T, Yokota S, Nakashima D, Higo M, Kasamatsu A, et al: Overexpression of LIM and SH3 protein 1 leading to accelerated G2/M phase transition contributes to enhanced tumourigenesis in oral cancer. PLoS One 8: e83187, 2013.

32. Iyoda M, Kasamatsu A, Ishigami T, Nakashima D, EndoSakamoto Y, Ogawara K, Shiiba M, Tanzawa H and Uzawa K: Epithelial cell transforming sequence 2 in human oral cancer. PLoS One 5: e14082, 2010.

33. Baba T, Sakamoto Y, Kasamatsu A, Minakawa Y, Yokota S, Higo M, Yokoe H, Ogawara K, Shiiba M, Tanzawa H, et al: Persephin: A potential key component in human oral cancer progression through the RET receptor tyrosine kinase-mitogenactivated protein kinase signaling pathway. Mol Carcinog 54: 608-617, 2013.

34. Minakawa Y, Kasamatsu A, Koike H, Higo M, Nakashima D, Kouzu Y, Sakamoto Y, Ogawara K, Shiiba M, Tanzawa H, et al: Kinesin family member $4 \mathrm{~A}$ : A potential predictor for progression of human oral cancer. PLoS One 8: e85951, 2013.

35. Lombardi DP, Geradts J, Foley JF, Chiao C, Lamb PW and Barrett JC: Loss of KAIl expression in the progression of colorectal cancer. Cancer Res 59: 5724-5731, 1999.

36. Shimada K, Uzawa K, Kato M, Endo Y, Shiiba M, Bukawa H, Yokoe H, Seki N and Tanzawa H: Aberrant expression of RAB1A in human tongue cancer. Br J Cancer 92: 1915-1921, 2005.

37. Kouzu Y, Uzawa K, Koike H, Saito K, Nakashima D, Higo M, Endo Y, Kasamatsu A, Shiiba M, Bukawa H, et al: Overexpression of stathmin in oral squamous-cell carcinoma: Correlation with tumour progression and poor prognosis. Br J Cancer 94: 717-723, 2006.

38. Maula S-M, Luukkaa M, Grénman R, Jackson D, Jalkanen S and Ristamäki R: Intratumoral lymphatics are essential for the metastatic spread and prognosis in squamous cell carcinomas of the head and neck region. Cancer Res 63: 1920-1926, 2003.

39. Kim SY, Nam SY, Choi SH, Cho KJ and Roh JL: Prognostic value of lymph node density in node-positive patients with oral squamous cell carcinoma. Ann Surg Oncol 18: 2310-2317, 2011.

40. Nicolson GL: Paracrine and autocrine growth mechanisms in tumor metastasis to specific sites with particular emphasis on brain and lung metastasis. Cancer Metastasis Rev 12: 325-343, 1993.

41. Thomas GJ, Lewis MP, Hart IR, Marshall JF and Speight PM: AlphaVbeta6 integrin promotes invasion of squamous carcinoma cells through up-regulation of matrix metalloproteinase-9. Int J Cancer 92: 641-650, 2001

42. Ylipalosaari M, Thomas GJ, Nystrom M, Salhimi S, Marshall JF, Huotari V, Tervahartiala T, Sorsa T and Salo T: $\alpha v \beta 6$ integrin down-regulates the MMP-13 expression in oral squamous cell carcinoma cells. Exp Cell Res 309: 273-283, 2005.

43. Ramos DM, But M, Regezi J, Schmidt BL, Atakilit A, Dang D, Ellis D, Jordan R and Li X: Expression of integrin $\beta 6$ enhances invasive behavior in oral squamous cell carcinoma. Matrix Biol 21: 297-307, 2002

44. Su C, Chen Z, Luo H, Su Y, Liu W, Cai L, Wang T, Lei Y and Zhong B: Different patterns of NF-kappaB and Notch1 signaling contribute to tumor-induced lymphangiogenesis of esophageal squamous cell carcinoma. J Exp Clin Cancer Res 30: 85, 2011. 